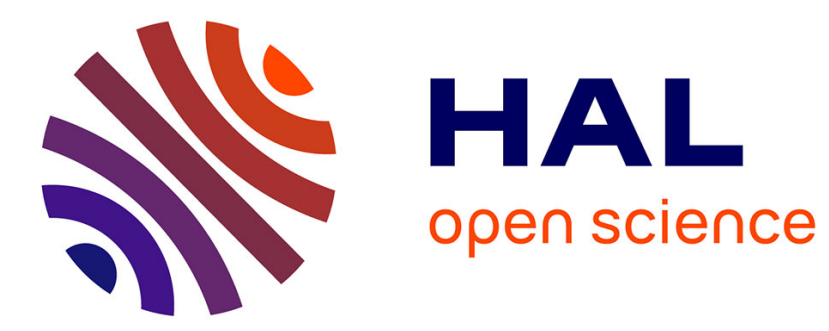

\title{
Modeling of segmented pure polyurethane electrostriction behaviors based on their nanostructural properties
}

M.H. Jomaa, Karine Masenelli-Varlot, G. Diguet, L. Seveyrat, L. Lebrun, K. Wongtimnoi, C. Véchambre, J.-M. Chenal, J.Y. Cavaille

\section{To cite this version:}

M.H. Jomaa, Karine Masenelli-Varlot, G. Diguet, L. Seveyrat, L. Lebrun, et al.. Modeling of segmented pure polyurethane electrostriction behaviors based on their nanostructural properties. Polymer, 2015, 62, pp.139-147. 10.1016/j.polymer.2015.02.016 . hal-01764871

\section{HAL Id: hal-01764871 \\ https://hal.science/hal-01764871}

Submitted on 14 Jun 2019

HAL is a multi-disciplinary open access archive for the deposit and dissemination of scientific research documents, whether they are published or not. The documents may come from teaching and research institutions in France or abroad, or from public or private research centers.
L'archive ouverte pluridisciplinaire HAL, est destinée au dépôt et à la diffusion de documents scientifiques de niveau recherche, publiés ou non, émanant des établissements d'enseignement et de recherche français ou étrangers, des laboratoires publics ou privés. 


\title{
Modeling of segmented pure polyurethane electrostriction
}

\section{behaviors based on their nanostructural properties}

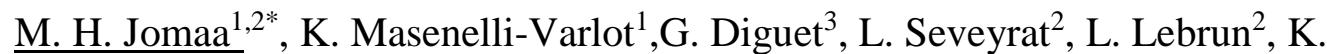
Wongtimnoi $^{1}$, C. Vechambre ${ }^{1}$, J. M. Chenal ${ }^{1}$, J.Y. Cavaillé ${ }^{1 .}$

${ }^{1}$ Université de Lyon, INSA-Lyon, CNRS, MATEIS, F-69621 Villeurbanne Cedex, France

${ }^{2}$ Université de Lyon, INSA-Lyon, LGEF, F-69621 Villeurbanne Cedex, France

${ }^{3}$ University of Johannesburg, Johannesburg PO Box 524 Auckland Park 2006 South Africa

*Author to whom all correspondence should be addressed

\begin{abstract}
Polyurethane (PU) exhibit very high electromechanical activity, and has a great interest for a wide range of transducer and actuator applications. It has been recently pointed out that this strong electrostriction may result from the phase separation. In the present work, a model taking account the PU nanostructure is presented. In order to validate this model, three PUs of similar compositions but different soft segment fractions have been characterized by DSC, TEM, AFM and SAXS and their electrostriction properties have been measured. When taking into account the diameter of the hard domains (HD) and the HD-HD distance, it is found that the model proposed well reproduces the experimental electrostriction properties of these phase-separated PUs.
\end{abstract}

Keywords: Polyurethane, electrostriction, modeling, microstructure.

\section{Introduction}

Electroactive Polymers (EAP) are a relatively new class of "smart material" that deform in the presence of an applied electric field [1], meaning they can convert electrical energy into mechanical energy [2]. EAPs can be used in various applications including actuators and 
sensors, biomimetics, robotics, energy harvesting and storage devices, among others [2, 3]. Polyurethanes have demonstrated their ability to convert electrical energy into mechanical energy and vice versa. Furthermore, these materials are lightweight, very flexible, blood and tissue compatible, highly resistant to abrasion; they present low manufacturing costs, and can be readily molded into any desirable shape [4]. Segmented polyurethane elastomers are block copolymers with alternating soft and hard blocks. In the present study the hard segments (HS) are composed of 4.4'-methylene-bis-(phenyl-isocyanate) (MDI) and 1,4-butanediol (BDO) and Poly(tetramethylene-oxide) is used as soft segments (SSs). The soft segments originate from the polyol and impart elastomeric characteristics to the polymeric material; the hard segments contain the highly polar urethane linkages and act as high modulus "fillers". Due to structural differences/incompatibility, microphases or domains can be formed from the respective hard and soft segments. Hard domains (HDs) - domains rich in HSs - play the role of physical crosslinks and act as high modulus fillers, whereas soft domains (SDs) - domains rich in SSs - provides extensibility [5,6]. The morphology of segmented PUs depends on the structure and relative amounts of these soft and hard domains and their ordering [7] (as well as from the form and the size distribution of HDs).

It is well documented that the properties and the performances of polyurethanes are strongly dependent on the degree of microphase separation and on the ensuing morphologies $[6,8]$. It has been recently pointed out that the strong electrostriction properties of pure segmented PU can be explained by the phase separation into HDs and SDs with high and weak dielectric constants, respectively. Indeed, phase separation leads to heterogeneities of both elastic and dielectric constants and to electric field gradients, which makes the SDs shrink and induces a compression of the whole material [9]. Nevertheless, this model is based on several microstructural parameters, such as the HD diameter, the HD-SD distance and the weight 
fractions of HDs and SDs. To our knowledge, the values of these parameters are still missing in the literature.

The purpose of this work is to investigate how the recent model can explain the electrostriction properties of segmented pure PUs. Since microstructural parameters are entry parameters of this model, an extensive microstructural characterization will be carried out. Three PUs with different weight fractions of HSs and SSs will be studied to check the robustness of the model.

\section{Material and methods}

Three different types of aromatic polyether-based thermoplastic PU are commercially available and were provided by the same company, Lubrizol, with different weight fractions of HSs and SSs. In the following, PU60, PU75 and PU88 respectively refer to Estane® ETE 60DT3 NAT022, Estane® X4977 NAT 039 and Estane® 58888 NAT021.From the supplier safety data sheet, it is mentioned that these PUs were synthesized by the same industrial process from the same initial chemical components. In addition to the different weight fractions of HSs and SSs, they also exhibit different molecular weights of SSs: 1000 (g/mol) for PU88 and PU60, and 2000 (g/mol) for PU75.

The polymer films were prepared by following a solution casting method. Before use, the PU granules were heated at $350 \mathrm{~K}$ for 3 hours. Then they were dissolved in N,Ndimethylformamide (DMF, Sigma-Aldrich D158550, 99\%) with a ratio of $25 \mathrm{wt} . \%$ of PU into DMF. The solution was heated at $350 \mathrm{~K}$ for 4 hours under mechanical agitation, until a homogeneous and viscous solution was obtained. Afterwards, this solution was casted on glass plates with an Elcometer 3700 doctor blade film applicator, put in an oven at $335 \mathrm{~K}$ for one day. A second heating treatment at $\mathrm{T}=400 \mathrm{~K}$ during 3 hours was then performed to eliminate any residual solvent. 
The microstructural morphology of segmented pure PU was studied by Differential Scanning Calorimetry (Setaram, DSC 131 Evo)) under nitrogen flow ( $\mathrm{P}=1.5 \mathrm{bar}$ ). Samples of $20 \mathrm{mg}$ were cooled to $140 \mathrm{~K}$ under liquid nitrogen and then heated to $480 \mathrm{~K}$ before being cooled to room temperature. The heating and cooling rates were set at $10 \mathrm{~K} / \mathrm{min}$.

Transmission Electron Microscopy (TEM) was used to investigate the phase separation into HDs and SDs but for that purpose, the samples had to be stained prior to the observation. The choice of the staining agent used in a given polymer system is guided largely by empirical success [10]. $\mathrm{OsO}_{4}$ and $\mathrm{RuO}_{4}$ are probably among the best known staining agents and are routinely used to stain polymers. $\mathrm{OsO}_{4}$ is believed to act as a covalent crosslinker between unsaturated carbon double bonds [11]. $\mathrm{RuO}_{4}$ is mainly used to differentially label aromatic moieties from aliphatic ones $[12,13]$. However due to similar chemical structures between HSs and SSs in the PUs studied here, a significant differential staining could not be obtained with $\mathrm{OsO}_{4}$ or $\mathrm{RuO}_{4}$ alone. Indeed for each PU type, a piece of film was stained in a $4 \%$ aqueous solution of $\mathrm{O}_{\mathrm{S}} \mathrm{O}_{4}$ for 24 hours. After rinsing with ethanol, the films of PU60 and PU75 turned to colors which clearly indicates incomplete staining see Fig. 1. $\mathrm{RuO}_{4}$ led to similar colors on the PUs studied here (not shown). Thus, a protocol similar to that of Janina Foks [14] was used, since they demonstrated that it was possible to localize the HDs of pure PU by using a double staining technique based on $\mathrm{OsO}_{4}$ and formaldehyde. By adding $\mathrm{OsO}_{4}$, Os stained double bonds on both segments as a first step. As a second step by adding formaldehyde and $\mathrm{OsO}_{4}$, formaldehyde opened the $\mathrm{N}-\mathrm{H}$ bonds and made it accessible for osmium (the $\mathrm{N}-\mathrm{H}$ bond exists only in the HS).

For each PU type, a piece of film was stained in a $4 \%$ aqueous solution of $\mathrm{OsO}_{4}$ for 24 hours. After rinsing with ethanol, the films were stained in a 50:50 aqueous solution of $\mathrm{O}_{\mathrm{S}} \mathrm{O}_{4}$ and $\mathrm{HCHO}_{4}$ (initial concentrations of $4 \%$ and $37 \%$ in water, respectively) for 24 hours [14]. After a second rinsing with ethanol, the films appeared to be completely black, which is an 
indication of a successful staining step (see Fig. 1). Thus, the films were embedded in an Epofix ${ }^{\circledR}$ resin and ultrathin sections were obtained by cryoultramicrotomy with a Reichert Ultracut S. The sample temperature was set to $200 \mathrm{~K}$ and the $35^{\circ}$ diamond knife speed to 1 $\mathrm{mm} / \mathrm{s}$.

TEM observations were carried out at acceleration voltage of $200 \mathrm{KV}$ with a JEOL 2010F microscope, operating at $200 \mathrm{kV}$. Bright field images were recorded with a Gatan Orius 200 camera. Measurements were performed manually using ImageJ on at least 90 objects.

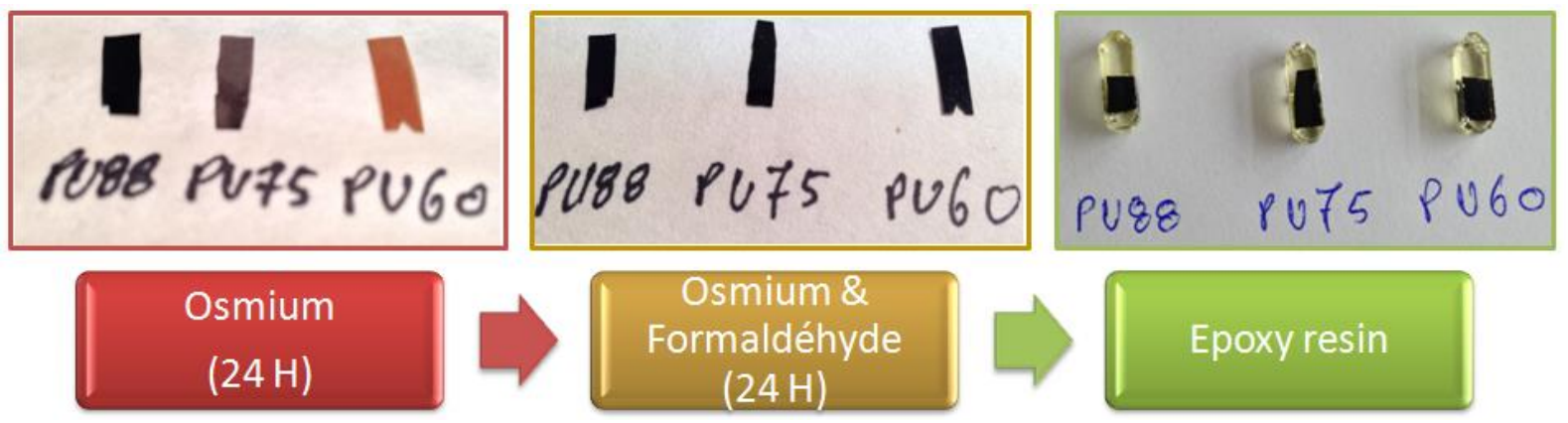

Fig. 1 Double staining protocol of segmented pure polyurethane.

Morphological observations of the HDs and SDs were performed by Atomic Force Microscopy (AFM) Nanoscope 5 (VEECO). Thin polymer films of each PU were deposed on a glass plate and dried for one night prior to imaging. Tapping mode phase images of sample surfaces were made using NanoProbe tips PPP-NCL (Nanosensor) for PU88 with a frequency of $153.2 \mathrm{kHz}$. PU75 and PU60 were analyzed with a NanoProbe tip SSS-NCH (Nanosensor) at a frequency of $272 \mathrm{kHz}$.

Small Angle X-ray Scattering (SAXS) was used to investigate the HD distribution. For that purpose, stacks composed of several layers of films were analyzed in transmission mode in two different directions, see Fig. 2. Patterns were acquired using a RU-300 generator equipped with a copper anode $\left(\lambda_{\mathrm{Cu}-\mathrm{K} \alpha}=1.54 \AA\right)$. The $1-2 \mathrm{~mm}$ thick stack was placed at a distance of $60 \mathrm{~cm}$ from the 2D CCD detector. 


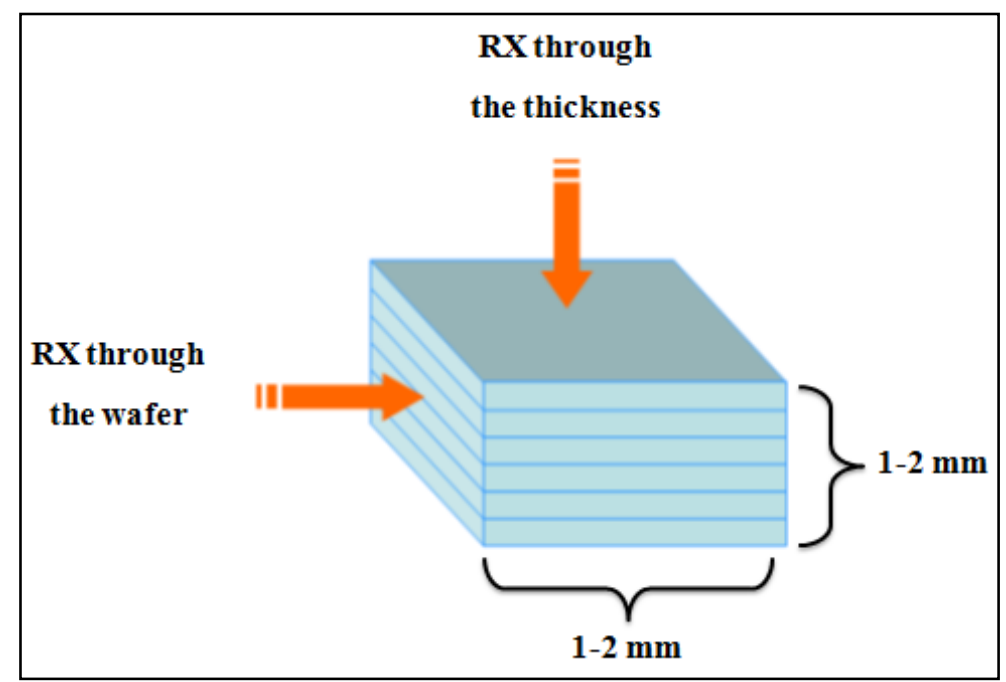

Fig. 2 Set-up used for SAXS characterisations. The samples are stacks composed of several layers of the films. Two different analysis directions are indicated.

The field-induced thickness strain Sexp was measured on circulars sample $(25 \mathrm{~mm}$ of diameter) with a homemade setup based on a double-beam laser interferometer measurement (Agilent 10889B) [15, 16]. Samples were placed between two cylindrical brass masses acting as conductive electrodes. A mirror was placed on the upper electrode to reflect the laser beam. A bipolar electric field was supplied by a high voltage amplifier (Trek10/10B) driven with a function generator (Agilent 33250A). Measurements were made at room temperature [17].

\section{Results and discussion}

\section{Modeling}

Guillot et al. explained from macroscopic level, that there was an empirical relationship between the electrostrictive coefficient, the dielectric constant and the compliance coefficient [18]. Recently, Diguet et al. pointed out that the phase separation into HDs with high dielectric constant and SDs with weak dielectric constant can result into the strong electrostriction properties of pure segmented PU. This, in turn, leads to heterogeneities of both elastic and dielectric constants and to electric field gradients, which makes the SDs 
shrink and the whole material compress [9]. In this work, Diguet's model is extended to take into account morphological parameters such as the HD diameter, the HD-SD distance and the HS weight fraction.

The strain is calculated from the Z-component of electric body force, created by HD taken as a sphere with radius $a$ into the surrounding SD. For a linear polarized dielectric medium in presence of an electric field $\boldsymbol{E}$, the Z-component of electric body force is:

$$
\vec{f}_{v_{-} z}=\left[-\frac{d}{d z}(-\vec{P} \cdot \vec{E})\right] \vec{u}_{z}=\left[\frac{d}{d z}\left(\varepsilon_{0} \chi \vec{E} \cdot \vec{E}\right)\right] \vec{u}_{z}=\left(\varepsilon_{S D}-\varepsilon_{0}\right) \frac{d E^{2}}{d z} \vec{u}_{z}
$$

Where $\varepsilon_{\mathrm{SD}}$ and $\chi$ are the dielectric constant and the electric susceptibility of the material respectively, $\varepsilon_{0}$ is vacuum permittivity and $\boldsymbol{P}$ is the material electric polarization.

Within a volume $V$ delimited by a surface $A_{S}$, the total force $\boldsymbol{F}$, the body force $\boldsymbol{f}_{\boldsymbol{v}}$ and the stress $\sigma$ are related as:

$$
\vec{F}=\int_{V} \vec{f}_{v} d V=\int_{A_{S}} \vec{\sigma} \cdot \vec{n} d A_{S}
$$

So, the force created at the distance $\mathrm{z} \geq \mathrm{a}$ from the centre of the sphere is obtained by summing contributions from the edge of a HD sphere:

$$
\int_{A_{S} a}^{z} \int_{a}^{z}\left(\varepsilon_{S D}-\varepsilon_{0}\right) \frac{d E^{2}}{d z} \vec{u}_{z} d z d A_{S}=\int_{A_{S}}\left(\varepsilon_{S D}-\varepsilon_{0}\right)\left(E^{2}(z)-E^{2}(a)\right) d A_{S}
$$

Identifying with (2), the stress is extracted from (3) and the deformation $S$ of the soft domain at the distance $\mathrm{z} \geq \mathrm{a}$ from the centre of the HD sphere is then obtained by dividing the stress by the SD Young modulus $Y_{\mathrm{SD}}$ :

$$
S(z)=\frac{\left(\varepsilon_{S D}-\varepsilon_{0}\right)\left(E^{2}(z)-E^{2}(a)\right)}{Y_{S D}}
$$


It can be seen that the strain is proportional to the difference of the square of the fields at the edge of the sphere and at the considered point. Consequently, the electric field inside the material has to be calculated. The applied electric field $E_{0}$ is considered to be directed along the $\mathrm{u}_{\mathrm{z}}$ direction. Using boundaries conditions and considering only the Z-component (1D), the electric field created out $(\mathrm{z} \geq \mathrm{a})$ of the dielectric sphere, can be expressed as:

$$
\vec{E}(z)=E_{0}\left[1-2 A\left(\frac{a}{|z|}\right)^{3}\right] \vec{u}_{z}
$$

With A the so-called dielectric contrast defined by:

$$
A=\frac{\varepsilon_{S D}-\varepsilon_{H D}}{2 \varepsilon_{S D}+\varepsilon_{H D}}
$$

The constant A varies, depending on the HD sphere and its SD surrounding dielectric constants, between -1 and 0.5 for $\varepsilon_{\mathrm{SD}} / \varepsilon_{\mathrm{HD}} \rightarrow 0$ and $\varepsilon_{\mathrm{HD}} / \varepsilon_{\mathrm{SD}} \rightarrow 0$ respectively.

Considering two spheres weakly interacting spaced by a distance $2 \mathrm{D}$, i.e. one placed at $\mathrm{z}=-\mathrm{D}$ and the second one placed at $\mathrm{z}=+\mathrm{D}$, the electric field is expressed as the superposition of the fields created by each of the sphere:

$$
\vec{E}(z)=\left[E_{0}-2 A E_{0}\left(\frac{a}{|z-D|}\right)^{3}-2 A E_{0}\left(\frac{a}{|z+D|}\right)^{3}\right] \vec{u}_{z}
$$

At the middle of the gap $(\mathrm{z}=0)$, the electric field is:

$$
\vec{E}(z=0)=E_{0}\left[1-4 A\left(\frac{a}{|D|}\right)^{3}\right] \vec{u}_{z}
$$

And far away from both spheres $(\mathrm{z}= \pm \infty)$, the electric field is: 
$\vec{E}(z= \pm \infty)=\vec{E}_{0}$

At the sphere interfaces $(|z|=D \pm a)$, the electric field is:

$$
\vec{E}(|z|=D \pm a)=E_{0}\left[1-2 A\left(1+\left(\frac{1}{2 \frac{D}{a} \pm 1}\right)^{3}\right)\right] \vec{u}_{z}
$$

As a quick estimation, with $\mathrm{A}=-3 / 4$ and $\mathrm{D}>>\mathrm{a}$, equations (8) and (10) give $\mathrm{E}(0)=\mathrm{E}_{0}$ and $\mathrm{E}(|\mathrm{z}|=\mathrm{D} \pm \mathrm{a})=2.5 \mathrm{E}_{0}$ which can be seen in Fig. 3; or to be more precise, with $\mathrm{A}=-3 / 4$ and $\mathrm{D}=4 \mathrm{a}$, $\mathrm{E}(0)=1.04 \mathrm{E}_{0}$ which can be seen in the inset of the figure below.

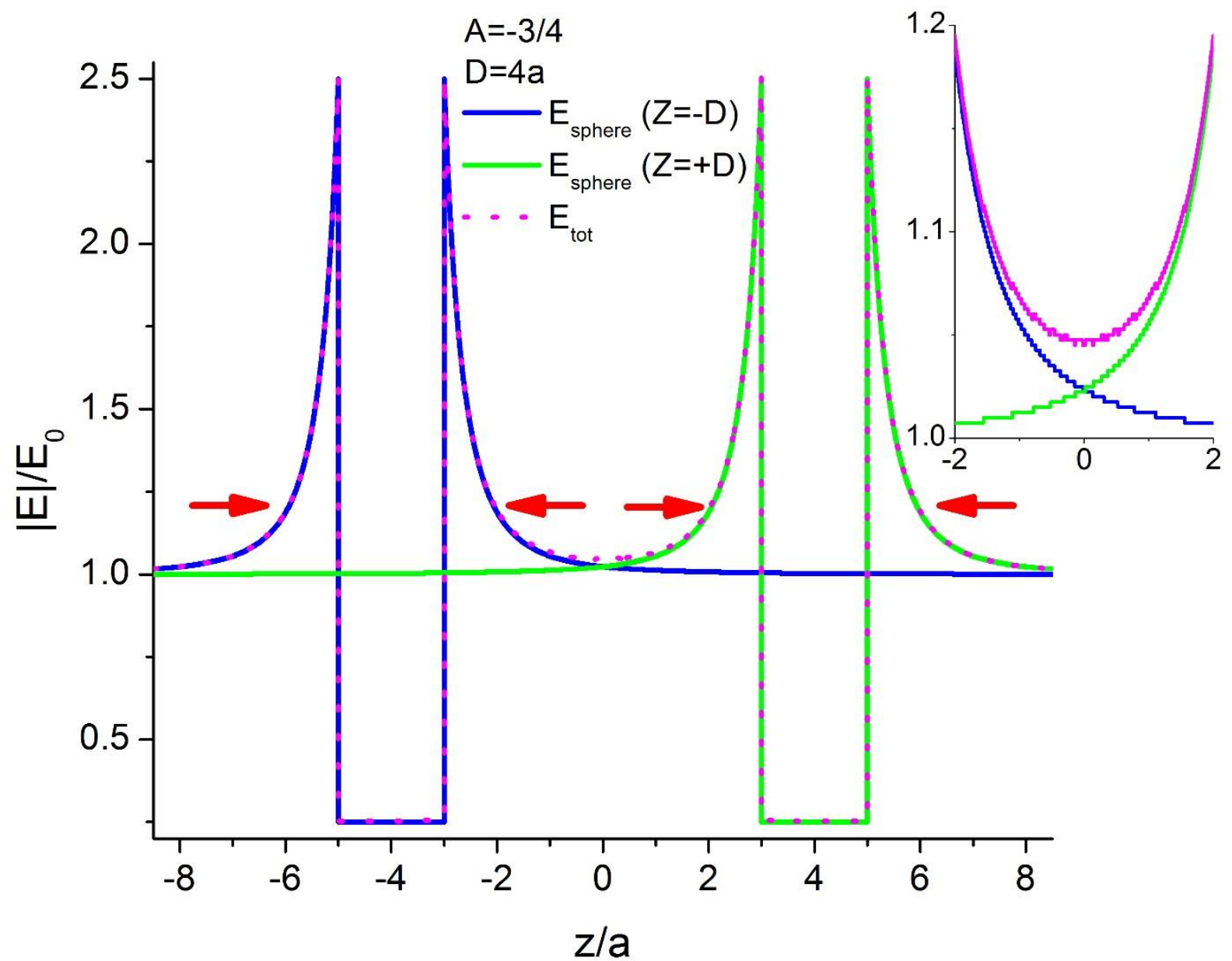

Fig. 3 : Value of the electric field calculated from equations (6) to (9), with a the sphere radius, the half distance between both spheres $D$ equal to 4 a and the dielectric contrast $A$ equal to $-3 / 4$. The inset shows a magnified view of the electric field variations in the gap between both spheres. 
As seen in Fig.3, there are 4 regions of SD deformation which can be reduced to 2 regions because of the symmetry; the region named below as 'inner' corresponded to the two deformations between the spheres whereas the region named below as 'outer' corresponded to the two deformations in the region apart the spheres.

The outer deformation can then be expressed from (4) with (9) and (10) as:

$$
\begin{aligned}
& S_{\text {outer }}=2 \frac{\left(\varepsilon_{S D}-\varepsilon_{0}\right)}{Y_{S D}}\left(E^{2}(|z|=\infty)-E^{2}((|z|=D+a))\right) \\
& S_{\text {outer }}=K\left(1-\left[1-2 A\left(1+\left(\frac{1}{2 \frac{D}{a}+1}\right)^{3}\right)\right]^{2}\right) \vec{u}_{z}
\end{aligned}
$$

Where $K=2 \frac{\left(\varepsilon_{S D}-\varepsilon_{0}\right)}{Y_{\text {soft }}}$,

And the inner deformation is expressed from (4) with (8) and (10) as:

$$
\begin{aligned}
& S_{\text {inner }}=2 \frac{\left(\varepsilon_{S D}-\varepsilon_{0}\right)}{Y_{S D}}\left(E^{2}(z=0)-E^{2}((|z|=D-a))\right) \\
& S_{\text {inner }}=K\left(\left[1-4 A\left(\frac{a}{|D|}\right)^{3}\right]^{2}-\left[1-2 A\left(1+\left(\frac{1}{2 \frac{D}{a}-1}\right)^{3}\right)\right]^{2} \vec{u}_{z}\right.
\end{aligned}
$$

For a long chain of spheres, the outer contribution acts only at the ends of the chain while the inner one acts at every gap between spheres. Hence, for long chain of spheres, the most 
contributive component is expected to be the inner contribution and outer contribution will be neglected below. The final strain is weighted as follow; first, the deformation between the HD is obtained by $S_{\text {inner }}=\frac{\Delta L_{S D}}{L_{S D}}$ meaning the SD change of length normalized by the SD initial length whereas the global deformation is given by $S_{t o t}=\frac{\Delta L}{L}$. Since $\Delta L_{S D}=\Delta L$ and $L_{S D}=L \phi_{S D}\left(\phi\right.$ the SD fraction) then we can write $S_{t o t}=\frac{\Delta L_{S D} \phi_{S D}}{L_{S D}}=S_{\text {inner }} \phi_{S D}$. The 1D SD fraction is $\phi_{S D}=\frac{2(D-a)}{2 D}=1-\frac{a}{D}$, and finally we can express the global deformation by :

$$
S_{\text {tot }}=S_{\text {inner }}\left(1-\frac{a}{D}\right)
$$

For a 3D system: $\phi_{H D}=\left(\frac{a}{D}\right)^{3}$, thus $\phi_{\mathrm{SD}}=1-\phi_{\mathrm{HD}}$. 

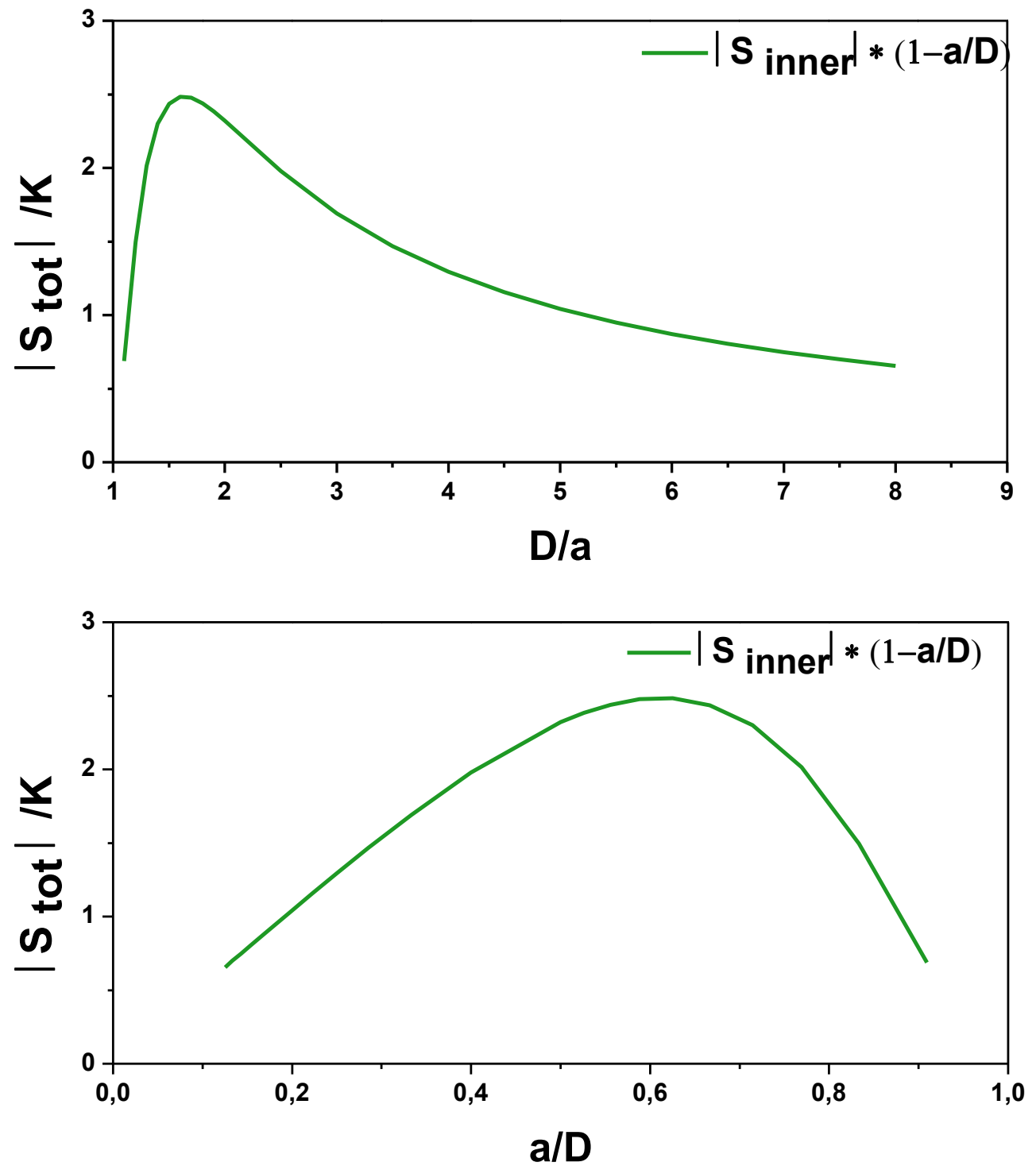

Fig. 4 : calculated variations of the total strain -1D system- for different values of a (sphere diameter) and $D$ (half-distance between two spheres). The dielectric contrast A was fixed to $\mathbf{- 3 / 4}$.

The variations of the total strain in function of the morphological parameters a and D are shown in

Fig. 4. It is noteworthy that the total strain is highly dependent on D/a. Thus, the experimental measurement of such parameters - as well as the shape of the HD - is crucial to validate the model.

2. Experimental determination of the morphological parameters

a. Phase separation 
The DSC thermograms presented in Fig. 5 and Fig. 6 were recorded in order to study the influence of the HS weight fraction as well as that of the phase separation on the crystalline morphology of the polyurethane host. The glass transition temperature for the soft segments ( $\left.T_{g S D}\right)$, melting temperatures $\left(T_{m_{I I} H D}\right.$ and $\left.T_{m_{I I I} H D}\right)$, heat of fusion $\left(\Delta H_{m}\right)$, crystallization temperature $\left(T_{c}\right)$ and heat of crystallization $\left(\Delta H_{c}\right)$ are summarized in Table 1.

Two temperature cycles were run in order to decouple the effects of the chemical structure and those due to the elaboration conditions. The first run $(\mathrm{C} 1)$ reflects PU microstructures partly due to their thermal history whereas before the second run $(\mathrm{C} 2)$, the thermal history was erased through cooling from the viscous melt. It can be noticed that the thermal events observed during the first run are always more visible than during the second one, especially around the HD melting temperature, $T_{m_{I I} H D}$ (not shown). This suggests that the time during which the PU chains can move is larger within the elaboration process involving a solvent than inside the DSC oven, and thus the phases are better organized. Furthermore we agree with a reviewer that this kind of thermal transition can be annealed out of the film with heat treatment. A thermal pretreatment is often used for polymers before DSC analysis. This pretreatment must be done at a temperature greater than $443-453 \mathrm{k}$ and it is a problem because it can imply a possible degradation of PUs [19]. For this reason the results given are based on the analysis of the first run. 


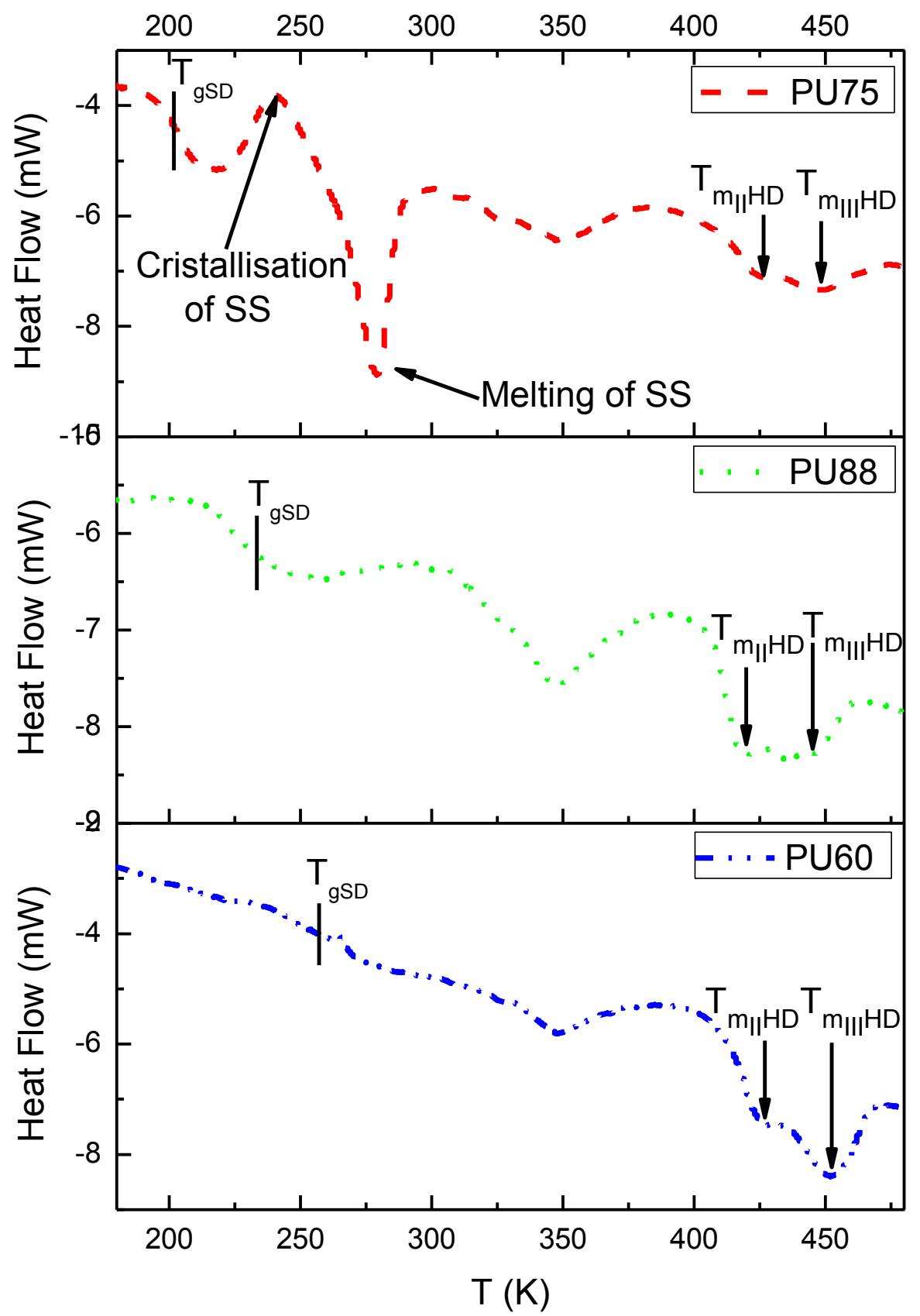

Fig. 5 DSC thermograms of the 3 pristine PUs upon heating $\left(1^{\text {st }}\right.$ run $)$ 
Table 1. Parameters extracted from the DSC thermograms for the 3 PUs. $T_{g S D}, T_{m_{I I} H D}, T_{m_{I I I} H D}$ are the glass transition temperatures related to the soft domains, and melting temperatures of hard domains, respectively. $T_{c}$ is the crystallization temperature. $\Delta H_{m}$ and $\Delta H_{c}$ and the enthalpies of fusion and crystallization, respectively.

\begin{tabular}{c|cccccc}
\hline \multicolumn{2}{c}{$\boldsymbol{T}_{\boldsymbol{g S D}}(\mathbf{k})$} & $\boldsymbol{T}_{\boldsymbol{m}_{\boldsymbol{I I}} \boldsymbol{H D}}(\mathbf{K})$ & $\boldsymbol{T}_{\boldsymbol{m}_{I I I} \boldsymbol{H D}}(\mathbf{K})$ & $\Delta \boldsymbol{H}_{\boldsymbol{m}}(\mathrm{J} / \mathrm{g})$ & $\boldsymbol{T}_{\boldsymbol{c}}(\mathbf{K})$ & $\Delta \boldsymbol{H}_{\boldsymbol{c}}(\mathrm{J} / \mathrm{g})$ \\
\hline PU75 & 205 & 425 & 447 & 11 & 285 & -17 \\
PU88 & 234 & 418 & 443 & 12 & 361 & -8 \\
PU60 & 259 & 427 & 451 & 16 & 423 & -15 \\
\hline
\end{tabular}

The glass transition temperature $T_{g S D}$ and the two melting temperatures suggest that each one of the 3 studied PUs has two "mixed" phases, one rich in SSs (the SDs), the other one rich in HSs (the HDs). This is in agreement with the results found by Saiani et al. [20]. The elaboration conditions (thermal history, evaporation of DMF, etc...) are important factors that influence the overall morphology of PUs.

The first thermal phenomena - near $204 \mathrm{~K}, 228 \mathrm{~K}$ and $254 \mathrm{~K}$ for PU75, PU88 and PU60 respectively- can be related to the glass transition of soft domains $\left(T_{g S D}\right)$. This value can give an estimation of the amount of hard segments (HS) dissolved in the SDs and as a consequence an indication of the "purity" of the soft phase [21, 22]. Indeed, an increase of the HS weight fraction led to a significant decrease of the $T_{g S D}$ [23]. This suggests an increase of the SS fraction in the HS when increasing the SS fraction.

For PU75 a characteristic peak around $240 \mathrm{~K}$ seems to correspond to the crystallization of SSs then followed by melting around $280 \mathrm{~K}$ (see Fig. 5), which is confirmed by dynamic mechanical measurement on the same sample (not shown). This indicates that during elaboration, the cooling was rapid enough to prevent crystallisation of the SSs.

Moreover, a bimodal endothermal transition can be observed for the 3 PUs on the DSC thermograms around 425 and $450 \mathrm{~K}$. From the literature, the existence of a bimodal peak 
could correspond to the fusion of crystalline hard segments with two different chain lengths [21, 24]. Alternatively, it can be related to rearrangements within the HDs and SDs, followed by the fusion of crystalline HDs $[25,19]$. In the two cases an increase of these temperatures values or an increase of the bimodal endotherm enthalpy indicates an higher cristallinity. PU60 presents the highest $\Delta H_{\boldsymbol{m}}$ value in agreement with the highest fraction of HD.

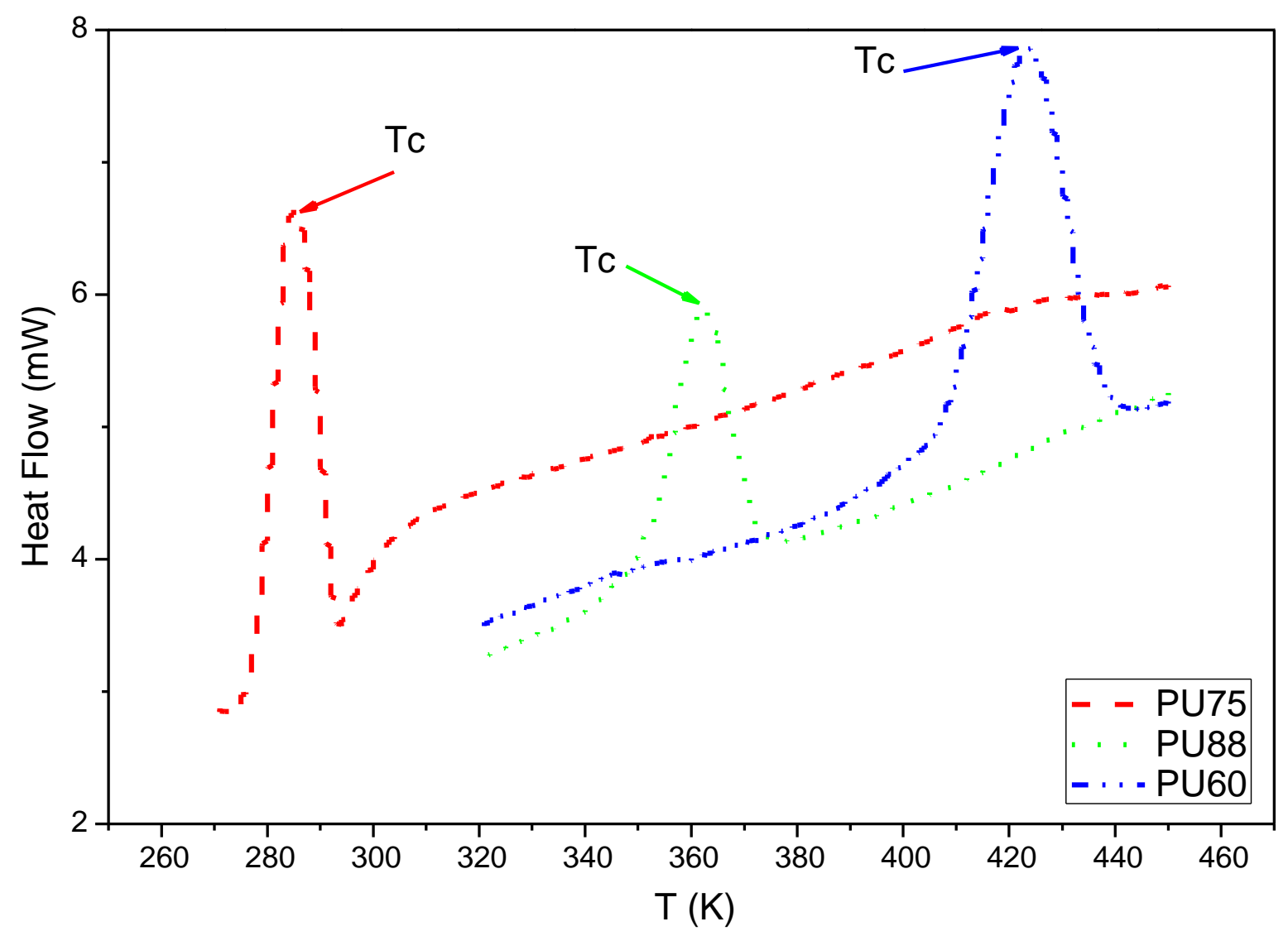

Fig. 6 DSC thermograms of the 3 pristine PUs upon cooling.

During cooling from the molten state to lower temperature (see Fig. 6), the 3 PUs exhibit a single exothermal crystallization event. This peak can be attributed to the crystallization of the HDs. Interestingly, the presence of one crystallization peak only does not seem to be compatible with 2 different HS lengths. This suggests that the bimodal peak observed upon heating was rather due to rearrangements of the HSs and SSs inside the HDs and SDs followed by melting of the HDs. The crystallization temperatures are highly dependent on the 
HS fraction (see Table 1). Indeed, they are equal to $285 \mathrm{~K}, 361 \mathrm{~K}$ and $423 \mathrm{~K}$ for PU75, PU88 and PU60, respectively. It is thought that an increase of the HS fraction leads to an enrichment of the HS fraction within the HDs, which facilitates their crystallization.

\section{b. Diameter distribution of HDs}

AFM exhibits a very high resolution for imaging morphologies at the surfaces of segmented copolymers $[26,27]$. In the tapping mode, the crystalline or hard domains in segmented copolymers can be imaged even at low hard-segment contents [28, 29]. Fig. 7 presents typical phase images obtained on PU75, PU88 and PU60 by AFM.
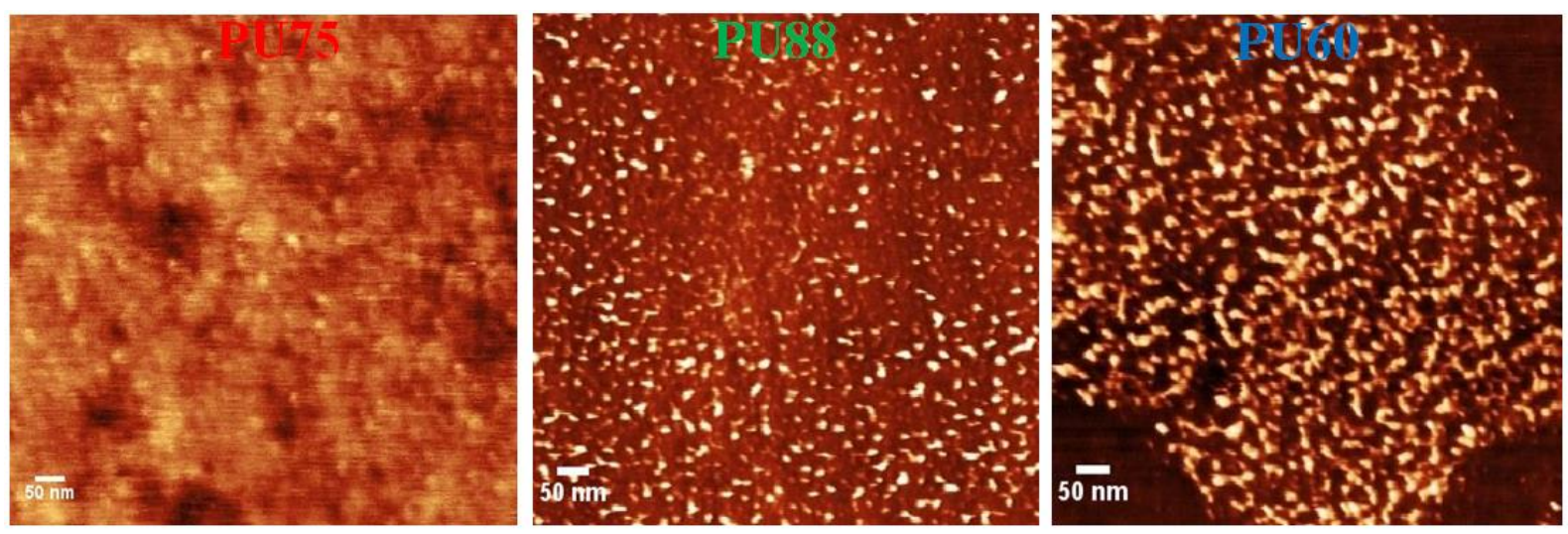

Fig. 7 AFM micrographs for PU75, PU88 and PU60 (phase images). The bright regions correspond to stiffer domains, i.e. the HDs.

These three segmented pure PUs exhibit two-phase morphologies, with bright stiff domains embedded in dark soft matrices $[30,31,32]$. This confirms the phase separation deduced from the DSC thermograms, the bright domains and the dark matrices being the HDs and the SDs, respectively. The HDs are found to be irregular in shape but mostly globular. A globular morphology is not always observed on polyurethane systems. Indeed, Elwell et al. evidenced percolated co-continuous microstructures [33]. Here, it is thought that the AFM images do not reveal co-continuous system. Indeed, the AFM images correspond to a planar section of the materials and in the case of co-continuity, elongated should lead to some elongated HDs. On 
the contrary, Schrader et al. [11] and Prici et al. [34] found a globular microstructure with a PU similar to the ones studied here, although with a different weight fraction of HS. This irregular shape and quite diffuse domain boundaries is thought to originate from polymer phase mixing in the form of hydrogen bonding between hard and soft regions of the polymer, which is commonly seen in polyether polyurethanes [30, 31].

The HD diameter distributions, measured from the AFM images, are presented in Fig. 9. The HD diameters mostly lie in the 6-14 $\mathrm{nm}$ range. It is noteworthy that the 3 PUs exhibit very similar HD diameter distributions. It can thus be concluded that the HS fraction has a negligible effect on the HD diameter but rather influences the HS content within each phase (HD and SD). The distribution of HD diameters can result on the one hand from an aggregation of different numbers of HSs and on the other hand from the existence of HSs of deferent sequence lengths [11].

A similar study was conducted by TEM on stained samples, to confirm the phase separation and the HD diameter distribution. Fig. 8 displays a representative micrograph obtained on PU75.

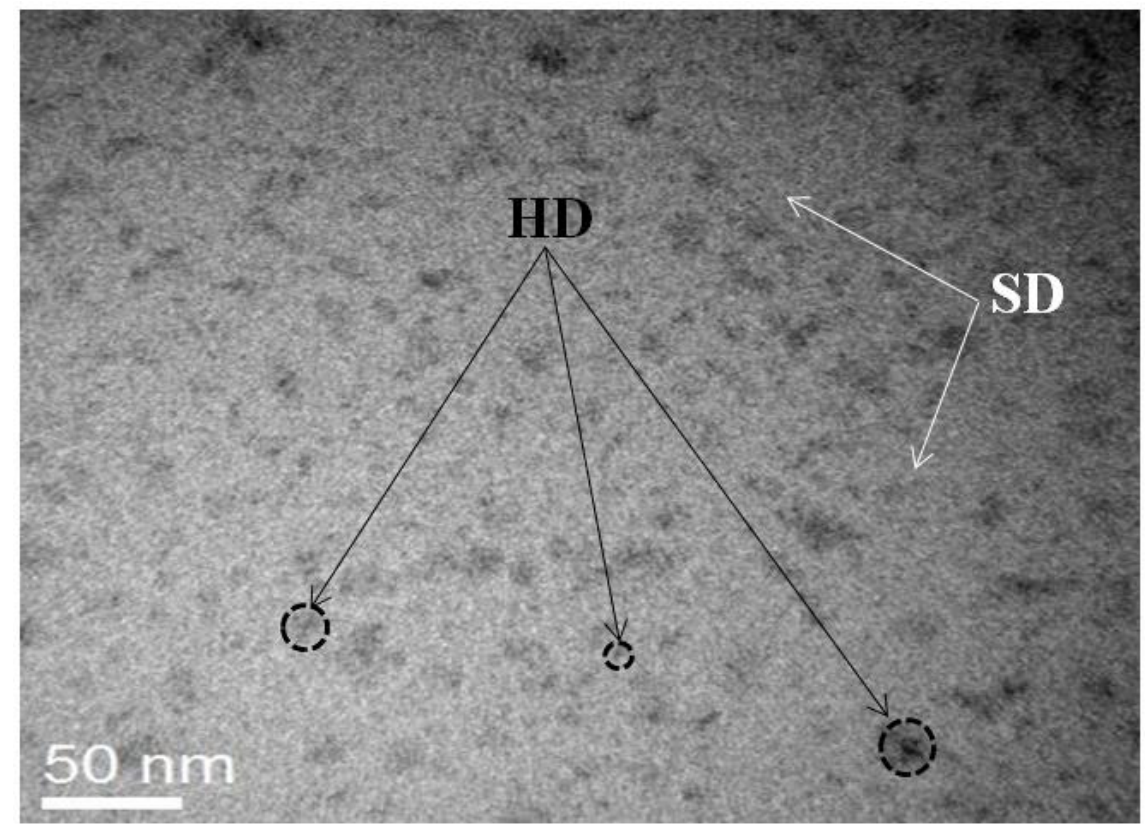

Fig. 8 TEM micrograph obtained on a stained sample of PU75. 
Again, two phases can be distinguished, with dark domains embedded in a bright matrix. As the double-staining method is expected to stain preferentially the hard segments, the dark domains can be attributed to the HDs and the bright matrix to the SDs. At this point, it is important to recall that TEM provides a $2 \mathrm{D}$ projected view of a $3 \mathrm{D}$ volume. Thus, the HDs may appear in contact with each other in the TEM image whereas they are disconnected and at different heights in the volume. Nevertheless, the microstructure observed by TEM is consistent with a globular morphology, in agreement with the results from AFM and from the literature [34].

The HD diameter distributions measured on TEM micrographs for each PU are presented in Fig. 9. For PU75 and PU88, the distributions are very similar to those derived from AFM, whereas the distribution is shifted towards smaller values for PU60. This shift is thought to be non-significant, since the distances measured on TEM bright field images are known to depend on the defocus [35]. Thus, it can be concluded that AFM and TEM lead to similar HD diameter distributions, and that the average HD diameter does not depend on the HS weight fraction. For the electrostriction model, a value of $10 \mathrm{~nm}$ can reasonably be used. 


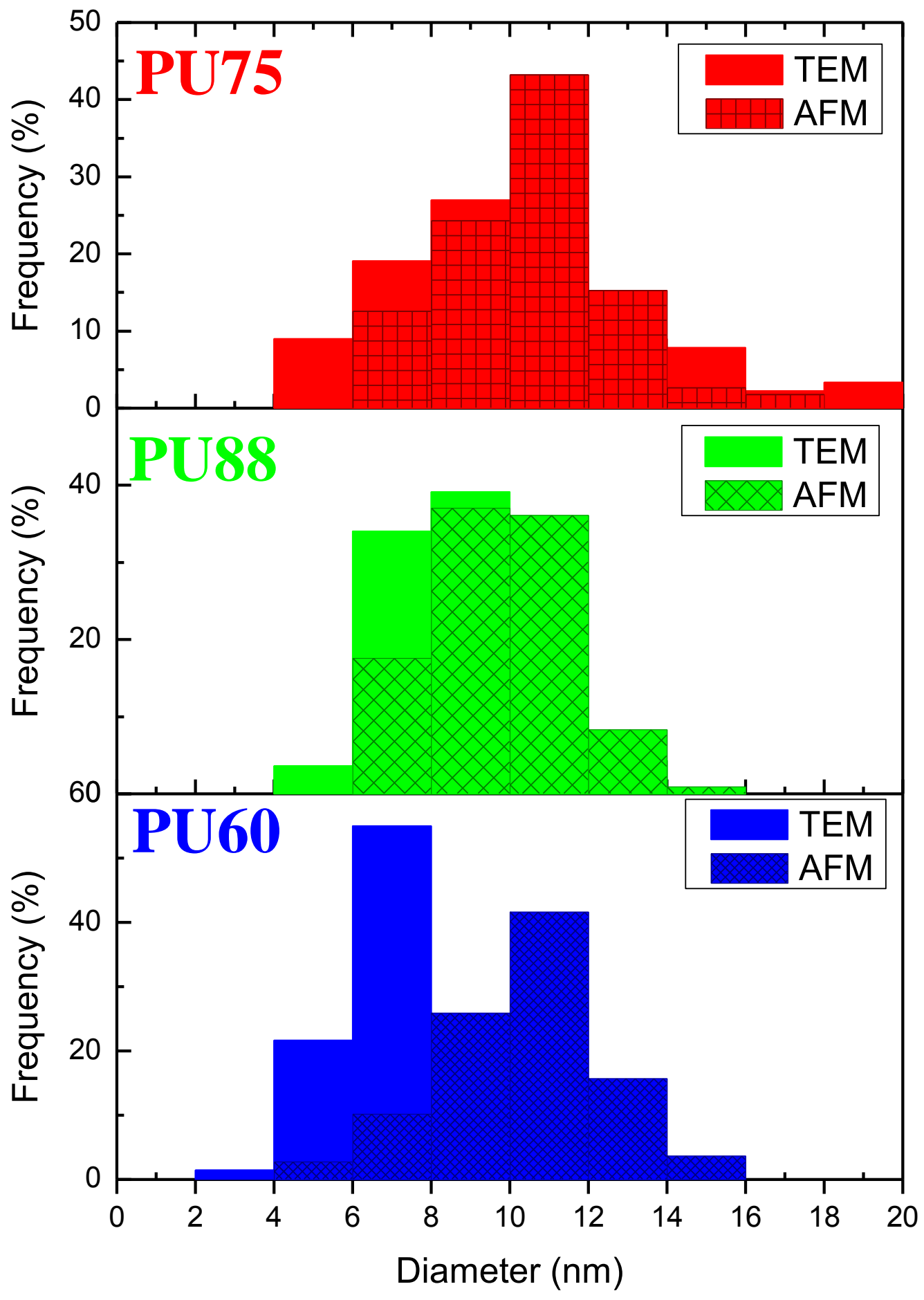

Fig. 9 Diameter distributions of hard domains of 3 PU using TEM and AFM microscopies.

c. Distance between HDs 
SAXS measurements were performed on the 3 PUs in order to measure the mean distance between HDs. The 2D SAXS patterns are shown in Fig. 10. A diffuse ring can be seen for each PU in each configuration. As the samples are obviously isotropic, the intensities were plotted in function of the scattering vectors q by averaging the signals over the rings.

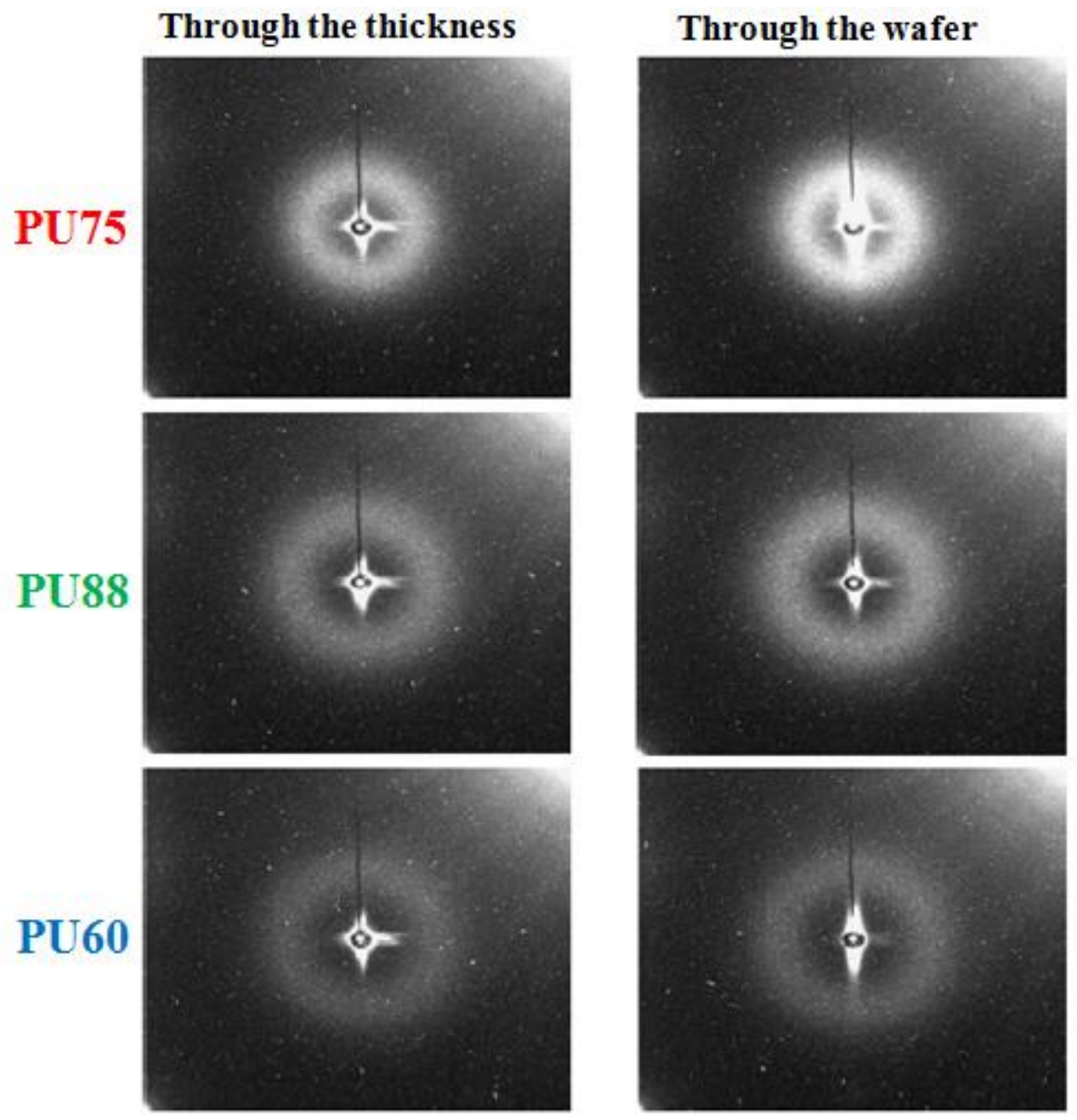

Fig. 10 SAXS 2D patterns obtained for the 3 PUs with two different orientations with respect to the X-ray beam.

The intensity variations in function of the scattering vector $\mathrm{q}$ are displayed in Fig. 11. The correlation bumps, corresponding to the rings observed on the $2 \mathrm{D}$ patterns, are clearly visible. From the previous analysis including DSC, AFM and TEM, the correlation bumps are expected to reflect the distribution of HDs in the film. The maxima of the correlation bumps 
can be used to determine a mean HD-HD distance, using Bragg relationship $d_{i}=\frac{2 \pi}{q_{\max }}[36]$.

The values are reported in Table 2. Clearly, the average distances between HDs lie in the 10$20 \mathrm{~nm}$. It decreases when increasing the HS fraction, as was qualitatively observed on the AFM images.

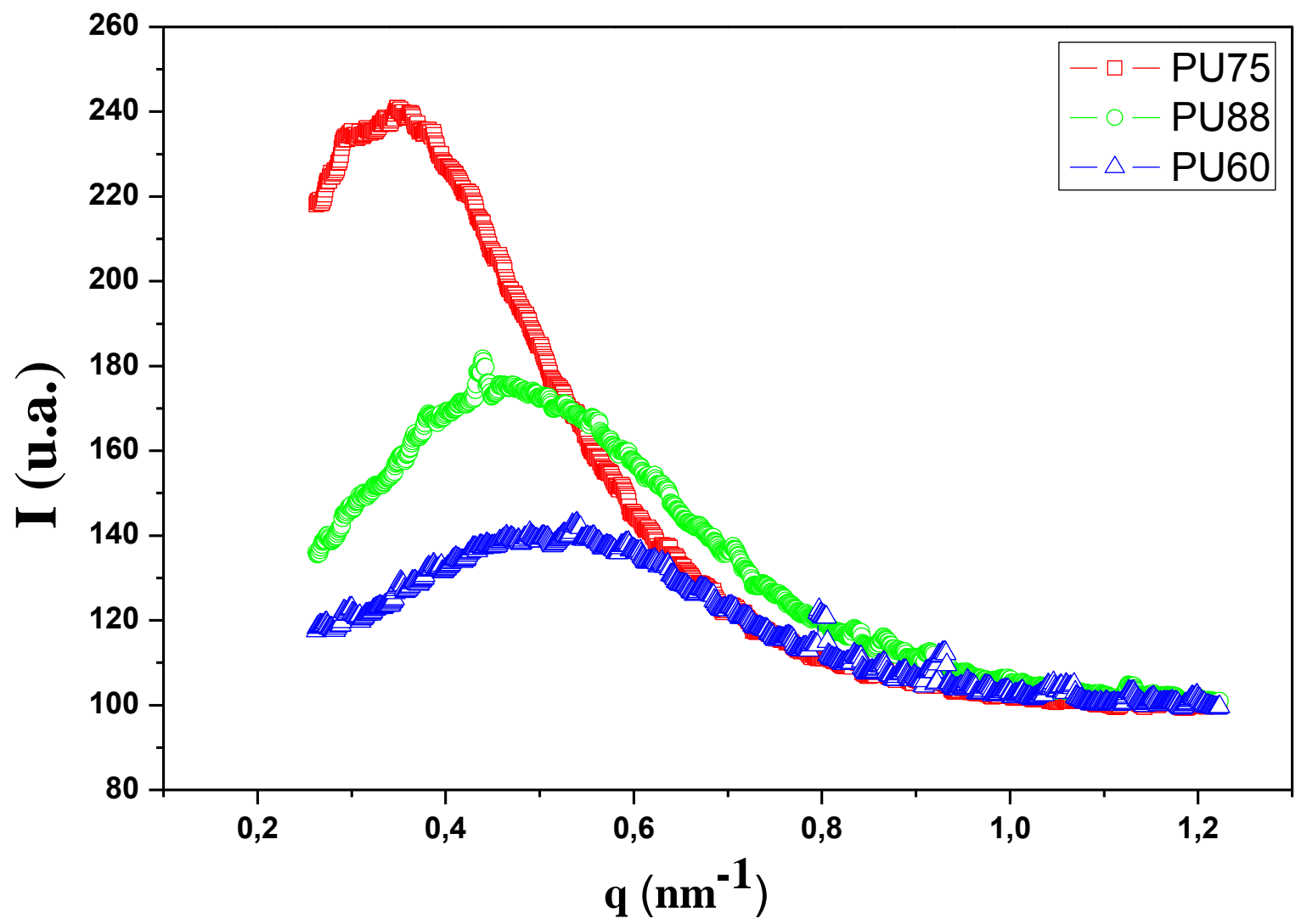

Fig. 11 Scattered intensity $I$ in function of the norm of the scattering vector $q$, for the 3 PUs.

Table 2. Average distance between two HD domains for the 3 PUs [23].

\begin{tabular}{c|ccc}
\hline & PU75 & PU88 & PU60 \\
\hline $\mathbf{q} \max \left(\mathbf{n m}^{-1}\right)$ & 0.36 & 0.44 & 0.53 \\
\hline Average distance $(\mathbf{n m})$ & 17.5 & 14.3 & 11.8 \\
\hline
\end{tabular}

\section{Electrostriction}


Based on the achieved parameters (diameter of HD -from TEM and AFM observation- and inter distance between HD and SD-from SAXS measurements) on the 3 pure segmented PUs, we calculated the inner and total deformations, either in $1 \mathrm{D}$ - considering only $\mathrm{Z}$ component, see equation (13)- or in 3D considering $(\mathrm{X}, \mathrm{Y}, \mathrm{Z})$ components. The values are presented in Table 3. For each PU, the experimental value, $S_{\text {exp }}$, measured using a laser interferometer setup, is given for comparison.

Table 3. Strain deformation of segmented PU; experimental \& calculated values

\begin{tabular}{|c|c|c|c|c|c|c|c|}
\hline & \multirow{2}{*}{\multicolumn{2}{|c|}{$1 \mathrm{D}$}} & \multirow{2}{*}{\multicolumn{2}{|c|}{$3 \mathrm{D}$}} & \multirow[b]{3}{*}{$\mathrm{S}_{\exp }(\%)$} \\
\hline & & & & & & & \\
\hline & $\overline{\mathbf{a}}$ & $\mathbf{D}$ & Sinner/K & $\mathbf{S}_{\text {tot }} / \mathbf{K}$ & S $_{\text {inner }} / \mathrm{K}$ & $\mathbf{S}_{\text {tot }} / \mathbf{K}$ & \\
\hline PU60 & 10 & 12 & 1.49 & 0.24 & 1.49 & 0.63 & 0.5 \\
\hline PU75 & 10 & 17 & 2.47 & 1.01 & 2.47 & 1.97 & 1.73 \\
\hline PU88 & 10 & 14 & 2.43 & 0.69 & 2.43 & 1.55 & 1.19 \\
\hline PU75/PU60 & & & & 4.08 & & 3.12 & 3.46 \\
\hline PU88/PU60 & & & & 2.79 & & 2.45 & 2.38 \\
\hline
\end{tabular}

There is a good correlation between experiments and model, especially with the 3D model. This validates the model based on the polymer nanostructure. This also suggests that the electrostriction properties of polyurethanes polymers can be tuned by controlling the polymer morphology, in terms of phase separation, HD diameter and HD-HD distance.

\section{Conclusions}

The purpose of this work was to provide a model of the segmented PU electrostriction properties, based on their microstructures and taking into account morphological parameters such as the HD diameter, the HD-HD distance and the HS weight fractions. Such model has been presented in a first part of the paper.

To validate the model, a complete analysis was performed by coupling DSC, TEM, AFM and SAXS experiments on three PUs with similar compositions but different weight fractions of 
HSs. It has been found that the 3 PUs exhibit segmented morphologies with spherical HDs embedded in soft matrices. The average HD diameter estimated from TEM and AFM observations has been found to be independent on the HS fraction since it was found to be equal to $10 \mathrm{~nm}$ in the three PUs. On the contrary, the HS fraction influences the distance between two adjacent HDs and may also play a role on the HS content inside the HDs and SDs. The distance was found to vary from $17 \mathrm{~nm}$ for the PU with the lowest fraction of HS to $12 \mathrm{~nm}$ for the PU with the highest fraction.

With the introduction of these microstructural parameters values into the models such as average HD diameter and HD-HD distance, the 3D model can well reproduce -with a good correlation- the experimental values of the electrostriction deformation, which validates the model. This opens the route to the design of materials with improved electrostrictive properties, by optimizing the diameter of HD and inter-distance HD-HD in order to improve and to have the best electrostrictive properties of segmented PU with globular morphologies.

\section{Acknowledgments}

The author acknowledges the French Agence Nationale de la Recherche (ANR) for financial support, under grant NAPOLECO (ANR- 2010-INTB-910-01), and the Centre Lyonnais de Microscopie (CLYM) for the access to the JEOL 2010F and Nanoscope 5 (VEECO) microscopes and the Centre Technologique des Microstructures for the access to the cryoultramicrotome. KMV would also like to thank B. Vastenhout and F. Gilleron for fruitful discussions regarding staining methods. 


\section{References}

[1] Bar-Cohen Y. Electroactive Polymer (EAP) Actuators as Artificial Muscles (Reality, Potential and Challenges). SPIE Press. Bellingham. 2004.

[2] Cathleen T ,Raghu DAS, Electroactive Polymers and Devices 2013-2018: Forecasts, Technologies, Players, Dielectric elastomers, electronic \& ionic EAPs and their applications

[3] Cottinet. P-J, Guyomar. D, Guiffard. B, Lebrun. L, Putson. C. Electrostrictive polymers as high-performance electroactive polymers for energy harvesting. INTEC. 2010. DOI: $10.5772 / 9946$

[4] Zokay S-P, Fergusont. J. Polyurethane elastomers. Prog. Polym. Sci.1991; 16: 695-836.

[5] Hepburn C. Polyurethane elastomers. 2nd ed. London: Elsevier; 1991.

[6] Petrović Z-S, Javni I. The effect of soft-segment length and concentration on phase separation in segmented polyurethanes. J. Polym.Sci. B Polym. Phys. 1989; 27: 545-560. DOI: 10.1002/polb.1989.090270305.

[7] Xu. Y, Petrovic. Z, Das. S, Garth. L-W. Morphology and properties of thermoplastic polyurethanes with dangling chains in ricinoleate-based soft segments. Polymer. 2008; 49: 4248-4258. DOI: 10.1016/j.polymer.2008.07.027

[8] Yang. C_Z., Grasel. T-G., Bell. J-L, Register. R-A, Cooper. S-L. Carboxylatecontaining chain-extended polyurethanes. J. Polym. Sci. Part B Polym. Phys. 1991; 28: 581-588.

[9] Diguet. G, Bogner. A, Chenal. J-M, Cavaillé. J-Y. Physical modeling of the electromechanical behavior of polar heterogeneous polymers, Journal of Applied Physics.2012; 112: 0000. DOI: 10.1063/1.4766280.

[10] Chou. T-M, Prayoonthong. P, Aitouchen. A, Libera. M. Nanoscale artifacs in RuO4stained poly(styrene). Polymer. 2002: 43; 2085-2088. DOI: 10.1016/S0032-3861(01)00767-4. 
[11] Schrader. S, Li. X, Guo. F, Liu. Y, Luo. J, Xu. D. Electron microscopy investigation of polyster-polyurethane elastromers stained with rutheinium teroxide. Die Makromolekulare Chemie, Rapid Communications.1998.; 9 : 597-601. DOI: 10.1002/marc.1988.030090901

[12] Haubruge. H-G, Jonas. A-M. Staining of poly(ethylene terephthalate) by ruthenium tetroxide. Polymer. 2003; 44: 3229-3234. DOI:10.1016/S0032-3861(03)00255-6

[13] Jeny I-S, Couchman. P-R. Ruthenium tetraoxide staining of polymers for electronmicroscopy. Macromolecules, 1983; 16: 589-598. DOI:10.1021/ma00238a021.

[14] Foks. J, Naumannz. I, Michler. G-H. beitrag zur aufklärung der morphologie von segmentierten polyurethanen/ contribution to the reconnaissance of the morphology of segmented polyurethanes. Die Angewandte MakromolekuIare Chemie. 1991; 189: 63- 76

[15] Guiffard B, Seveyrat L, Sebald G, Guyomar D. Enhanced electric field induced strain in non percolative carbon nanopower / polyurethane composites. J. of Phys. D: Appl. Phys. 2006; 3039- 3053.

[16] Guyomar D, Cottinet. P-J, Lebrun. L, Putson. C, Yuse. K, Kanda. M, Nishi. Y. The compressive electrical field electrostrictive coefficient M33 of electroactive polymer composites and its saturation versus electrical field, polymer thickness, frequency, and fillers. Polymers for Advanced Technologies. 2012; 23, 946-950. DOI: 10.1002/pat.1993

[17] Seveyrat. L, Guyomar. D, Lebrun. L. Preparation of graphene nanoflakes/polymer composites and their performances for actuation and energy harvesting applications. Journal of Applied Physics. 2012; 111. DOI. 10.1063/1.4718577.

[18] Guillot F M , Balizer E. Electrostrictive effect in polyurethanes. Journal of Applied Polymer Science. 2003; 89: 399-404. DOI: 10.1002/app.12096.

[19] Lapprand A, Mechin F, Pascault J-P. Synthesis and properties of self-crosslinkable thermoplastic polyurethanes.J. Appl. Polym. Sci. 2007; 105: 99-113. DOI: 10.1002/app.26086. 
[20] Saiani. A, Daunch. W-A, Verbeke. H, Leenslag. J-W, Higgins. J-S. Origin of Multiple Melting Endotherms in a High Hard Block Content Polyurethane. 1. Thermodynamic Investigation. Macromolecules. 2001; 34: 9059-9068

[21] Martin. D-J, Meijs. G-F, Gunatillake. P-A, McCarthy. S-J, Renwick. G-M. The effect of average soft segment length on morphology and properties of a series of polyurethane elastomers. II. SAXS-DSC annealing study. Journal of Applied Polymer Science. 1997; 64: 803. DOI: 10.1002/(SICI)1097-4628(19970425)64:4<803::AID-APP20>3.0.CO;2-T

[22] Chen. K-S, Leon. T-Y, Chen. Y-S, Lin. T-L, Liu. W-J. Soft- and hard-segment phase segregation of polyester-based polyurethane. Journal of Polymer Research. 2001; 8: 99. DOI: 0.1007/s10965-006-0139-3.

[23] Wongtimnoi. K, "Polyuréthanes électrostrictifs et Nanocomposites: Caractérisation et Analyse des mécanismes de couplages électromécaniques", PhD thesis, 2011, INSA-Lyon, France.

[24] Król. P, Pilch. B-P. Phase structure and thermal stability of crosslinked polyurethane elastomers based on well-defined prepolymers. Journal of Applied Polymer Science. 2007; 104: 1464. DOI: 10.1002/app.25011

[25] Koberstein J-T, Russell T-P. Simultaneous SAXS-DSC Study of Multiple Endothermic Behavior in Polyether-based Polyurethane Block Copolymers. Macromolecules. 1986; 19: 714-720. DOI: 10.1021/ma00157a039.

[26] McLean. S, Sauer. B-B. Nano-deformation of crystalline domains during tensile stretching studied by atomic force microscopy.J Polym Sci Part B: PolymPhys. 1999; 37: 859-866. DOI: 10.1002/(SICI)1099-0488(19990415)37:8<859::AID-POLB11>3.0.CO;2-U.

[27] McLean. S, Sauer. B-B. Tapping-mode AFM studies using phase detection for resolution of nanophases insegmented polyurethanes and other block copolymers. Macromolecules. 1997; 30: 8314-8317. DOI: 10.1021/ma970350e. 
[28] Brayan. B-S,Scott Maclean. R, Reinoud. J-G, Meike. C, Nilesten. J-E. Crystalline Morphologies in Segmented Copolymers with Hard Segments of Uniform Length. Journal of Polymer Science: Part B: Polymer Physics. 2004; 42: 1783-1792. DOI: 10.1002/polb.20060.

[29] Sauer. B-B, McLean. R-S, Brill. D-J. Londono. D-J. Morphology and orientation during the deformation of segmented elastomers studied with small-angle X-ray scattering and atomic force microscopy. J Polym Sci Part B: Polym Phys. 2002; 40: 1727-1740. DOI: $10.1002 /$ polb.10234

[30] Matithew. J-O, Bruce. D-L, Garth. L-W. Structure-Property Relationships of Poly(urethane urea)s with Ultra-low Monol Content Poly(propylene glycol) Soft Segments. I. Influence of Soft Segment Molecular Weight and Hard Segment Content. Journal of Applied Polymer Science. 2002; 84: 229-243. DOI: 0.1002/app.10168.

[31] Waletzko. R-S, LaShanda T. Korley. J, Brian D-P, Edwin L-T, Hammond. P-T. Role of Increased Crystallinity in Deformation-Induced Structure of Segmented Thermoplastic Polyurethane Elastomers with PEO and PEO-PPO-PEO Soft Segments and HDI Hard Segments. Macromolecules. 2009; 42: 2041-2053. DOI: 10.1021/ma8022052.

[32] Gogolewski, S. Selected Topics in Biomedical Polyurethanes- a Review. Colloid Polym. Sci. 1989; 267: 757-785.

[33] Michael J. Elwell, Anthony J. Ryan, Henri J. M. Gru nbauer and Henry C. Van Lieshout. In-Situ Studies of Structure Development during the Reactive Processing of Model Flexible Polyurethane Foam Systems Using FT-IR Spectroscopy, Synchrotron SAXS, and Rheology. Macromolecules. 1996; 29: 2960-2968.DOI:10.1021/ma9511208.

[34] Princi. E, Vicini. S, Stagnaro. P, Conzatti. L. The nanostructured morphology of linear polyurethanes observed by transmission electron microscopy. Micron. 2011; 42: 3-7. DOI:10.1016/j.micron.2010.09.007 
[35] Yao. B, Edwards. D-J, Kurtz. R-J, Odette. G-R, Yamamoto. T. Multislice simulation of transmission electron microscopy imaging of helium bubbles in Fe. J. Electron Microsc. 2012; 61: 393-400. Doi: 10.1093/jmicro/dfs065

[36] Saiani. A, Rochas. C, Eeckhaut. G, Daunch. W-A, Leenslag. J-W, Higgins. J-S. Origin of Multiple Melting Endotherms in a High Hard Block Content Polyurethane. 2. Structure Investigation. Macromolecules, vol. 37, p. 1411-1421, 2004. DOI: 10.1021/ma0105993. 